\title{
Two Web Resources Linking Major Human Embryology Collections Worldwide
}

\author{
Mark A. Hill \\ Anatomy, School of Medical Sciences, UNSW Sydney, Sydney, NSW, Australia
}

\section{Keywords}

Human embryology · Human development · Embryology education · Morphology · Histology

\begin{abstract}
Human embryology is a core subject for medicine and current research. While animal models of development now have significant online resources available, the vast majority of human embryonic material is locked up in historic collections. When accessed today, these collections are still contributing to our understanding of human development. This paper describes two online resources for studying human development that are unlocking these invaluable collections and providing related human developmental resources. The first of these is the online Embryology website (http:// tiny.cc/Embryo) that links the human developmental timeline to historic and current research findings. Secondly is the Digital Embryology Consortium (https://human-embryology.org), an international research partnership to digitise, preserve, and make the major embryology histological collections available for researchers. By making this histological material more widely available to researchers with new methods of analysis, a better understanding of human development can be reached. This also opens the opportunity for new $3 \mathrm{D}$ reconstruction and virtual reality representation of these embryos.

(c) 2019 S. Karger AG, Basel
\end{abstract}

(C) 2019 S. Karger AG, Basel

\section{Introduction}

Human embryology is a core subject for medicine and developmental biology as well as a major current research topic. Embryology is also a medical education subject that has seen a substantial decrease in contact hours in recent years [Drake et al., 2009]. There has been a paucity of well-designed online material presented in a way that students and researchers can more easily understand this topic. In contrast, there exist many online resources for a range of animal developmental and disease models, although human development is generally not a focus of these sites. While current medical research focusses on detailed disease genetics and molecular mechanisms, these also need be placed in the broader context of an understanding of the events in human developmental anatomy. Some examples of other research areas that would benefit from an improved understanding of key human developmental features are: congenital abnormalities, teratogenic effects during critical periods, stem cell differentiation, assisted reproductive technologies, and the developmental origins of heath and disease.

This paper will discuss the available web resources for human embryology, focussing on the human Embryology website (http://tiny.cc/Embryo) and a second research resource being developed by the Digital Embryology Consortium (DEC, https://human-embryology.org). The

\section{KARGER}

E-Mail karger@karger.com

www.karger.com/cto
Dr. Mark Hill

Anatomy, School of Medical Sciences, UNSW Australia

Room 221, Level 2, Wallace Wurth West

Sydney, NSW 2052 (Australia)

E-Mailm.hill@unsw.edu.au 
paper includes some weblinks to online resources that use TinyCC shortening of the original web addresses.

The purpose of the online Embryology website for the last 20 years has been to deliver readily available access to human embryology from the latest current research findings extending back through key historic discoveries. As most current embryology research employs only animal models, animal comparative data has also been integrated into the resource. Researchers should note though that animal models have some limitations for specific aspects of human development. The main animal models of development (mouse, rat, zebrafish, chicken, drosophila) have today substantially more online research and educational resources available than human development. Some of these animal resources can be found from this start page (http://tiny.cc/Animal_Development) for each species, and the online human site also provides a comparative timeline of animal model development to human development.

The author also acknowledges that in the last 10 years a number of additional excellent online co-ordinated human education resources have been developed (Virtual Human Embryo [HEIRLOOM], Embryology.ch, Embryo Project Encyclopedia, and Developmental Atlas [Yamada et al., 2010]) and numerous online educational videos of variable quality. Recently, there have been similar off-line resources published on human embryology [Hill, 2007] using both PDF [de Bakker et al., 2012, 2016] and eBook formats [Hill, 2016, 2018].

\section{Online History}

The first web browsers were developed between 1993 and 1995: Mosaic, Netscape Navigator, and Internet Explorer. These three initial web browsers have all been replaced over time by a range of new web browsers, which all do pretty much the same thing as the originals. In January 1997, PubMed went online; previous to this time the published research database was only available from university and other institution libraries by subscription. In 1998 Google search became available, and the era of rapid online searches began. Three years later, in 2001, Wikipedia went online, providing an online encyclopaedia populated with data provided by a variable quality of authors. For students today, both Google search and Wikipedia remain the "go to" resources for online searches. For researchers, PubMed, with about 1 million new papers each year, remains their "go to" resource. Excluding private companies and institutional research databases this involves over 1,200 petabytes of data. A brief developmental history of the online resource is given below, including a description of how the current human embryology content is organised by topic and also the standardised internal organisation of the topic page.

\section{Embryology Resource History}

In 1997 the human Embryology website (http://tiny. cc/Embryo) was first developed with the new internet. The original intranet site provided local students with access to histological serial cross-section images of two stages of embryonic development (Carnegie stage 13 and stage 22) and selected high-power images of the stage 22 embryo. These serial sections were originally developed and used in the 1970s to 1990s for local student viewing and human embryology teaching using a microfiche reader. The online development was aided by many medical students contributing to the generation of labelled versions of these histology images for their own educational purposes. To allow wider student access outside class hours, the content was then released annually, first as CD and later DVD versions to students and National Library holdings (see National Library of Australia holding https://trove.nla.gov.au/version/28976584).

As internet speeds developed the content was opened up as an internet website, allowing external user access. An early collaboration began with Prof. Kohei Shiota, from the Kyoto collection, enabling the display of surface views of human embryos from Carnegie stage 7 to 23; these were also displayed as scaled images to show the overall relative growth in size of the embryo during the first 8 weeks of development. This collaboration with the Kyoto collection, the largest collection of human embryos in the world, has been ongoing, and continues today with Prof. Shigehito Yamada. This online collaboration with embryology researchers was a key focus, and currently includes more than 50 contributing researchers. As the online content grew from the many external and internal contributions, difficulties navigating the existing and growing website increased.

In 2004, as part of a UNSW Innovative Teaching and Educational Technology 4 (ITET4) educational fellowship and associated with a redesign of the university medical program, the author completely redesigned the existing website in layout and navigation on all pages. One of the major improvements was the development of classspecific pages for both Science and Medical students, a new glossary of terms, external Google site search, and the 


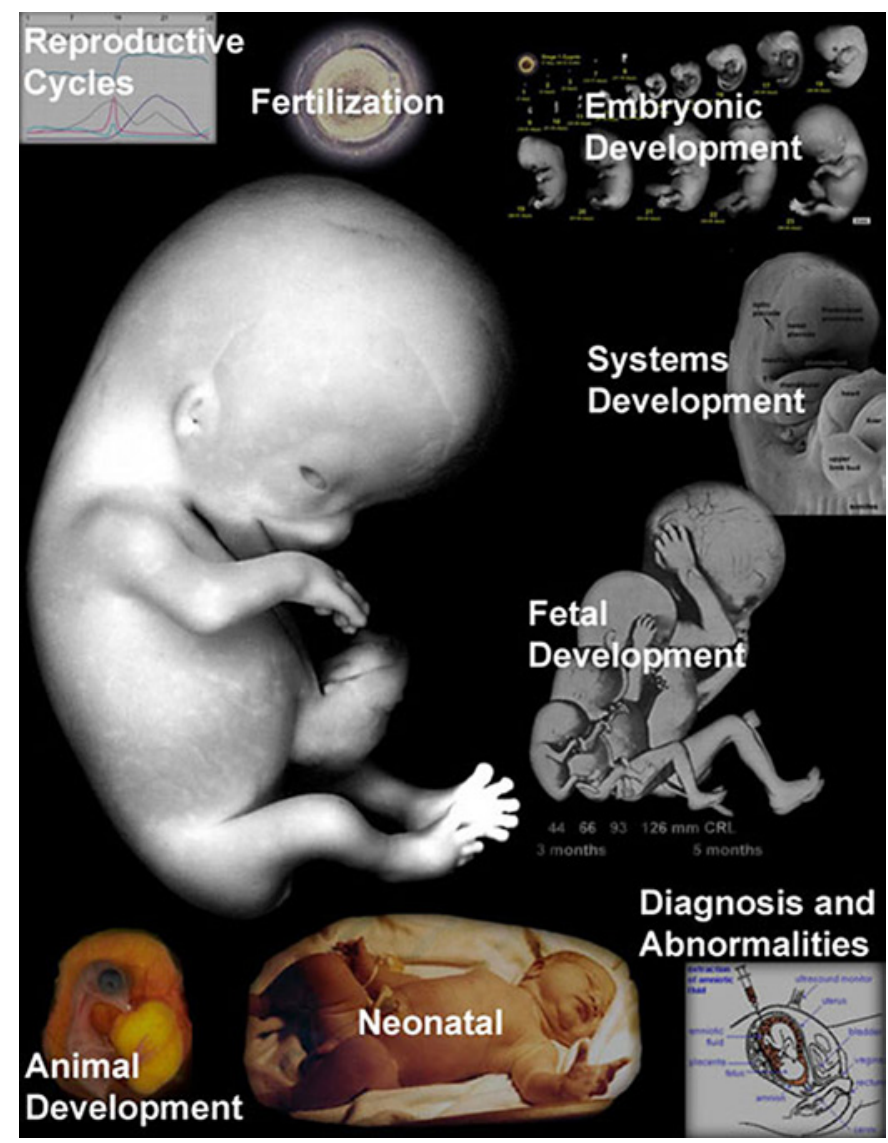

Fig. 1. Embryology website (http://tiny.cc/Embryo) opening page image showing major topics related to human development. When moused over the online image version links directly to the named topics.

addition of many educational and research animations/ movies. This online format worked successfully for many years, with embryonic data from the site compiled and published in 2007 [Hill, 2007].

In 2009, the website was completely redesigned to take advantage of the technical advances in the online environment (Fig. 1). The Mediawiki format was selected and required moving and rewriting the existing hundreds of pages, movies, and thousands of images into this new design. Mediawiki software had allowed the development of Wikipedia in 2001 and is a free software open source wiki package written in PHP. While the software is the same, the current page layout minimizes the navigation menu to the top of the screen, which also maximises the page content to the full screen width. There were a number of advantages of the Mediawiki software over the existing HTML website. Firstly, the ability to allow collaborative

Two Web Resources Linking Major Human Embryology Collections Worldwide contributions rather than from a single or few editor(s) contributions. Secondly, the navigation and page format were well known, and featured the ability to search the local site. Thirdly, the addition of categories of content, the equivalent of visible meta tags, allowed direct linking and the relationship of related pages and media. Fourthly, a large number of contributed extensions with important applications were introduced, such as: security log in, video embedding, image mapping, PubMed reference linking, quizzes, gadgets, and Google analytics integration. Finally, the ability for students and researchers to create accounts to make and edit content further extended the educational function of the website. Statistically, Google analytics reports show that each week the site currently logs more than 40,000 worldwide user sessions.

\section{Human Embryo Staging}

A consideration when studying human embryology, and found only with our species, is the timing difference between "fertilisation age" and "gestational age." All other species, without exception, begin development and staging at fertilisation, the "fertilisation age." In contrast, clinical medicine bases human development (pregnancy) on the arbitrary first day of the last menstrual period, the "gestational age." This is an easier to identify clinical marker, the time when the woman was not pregnant, and corresponds to about two weeks before fertilisation. This is so ingrained in clinical literature that modern assisted reproductive technology techniques still add two weeks to the actual date of known laboratory fertilisation. Rather than argue the point from an embryological view, both ages are generally included in the timing, with gestational age shown as "GA weeks."

The website uses week by week as well as the classical human embryonic 23 Carnegie "stages" or "horizons" (http://tiny.cc/Carnegie_Collection) developed by Mall and Streeter at the Carnegie Institute. These stages were based upon human embryos held by the Carnegie collection (http://tiny.cc/HDAC_Collections). This staging system has been used extensively in human embryo textbooks and the research literature. The same staging system has been used to classify human embryos held in the other large collections, most notably the Blechschmidt and Kyoto collections.

Each online Carnegie stage has its own page, for example see Carnegie stage 13 (http://tiny.cc/Carnegie_ Stage_13). The page features some or all of the following analyses where available: external embryo views, virtual 
slides, bright field microscopy, electron microscopy, histology, 3D reconstructions, related 3D magnetic resonance imaging (MRI), and episcopic fluorescence image capturing (EFIC; http://tiny.cc/Stage_13_EFIC_1) analysis movies [Dhanantwari et al., 2009; Krishnan et al., 2014]. Virtual slides describe online embryo (http://tiny.cc/Virtual_ Slide_CS13) and histology images that have been stored in a format that allows viewing at a range of magnifications, for example see the stage 22 histology screenshot (Fig. 2). These stage resource pages provide detailed embryo viewing modalities that allow identification of the external and internal appearance, anatomy, and examples from the various collections. Information on key stage developmental events is also linked directly to the published literature available online or within the PubMed database.

\section{Human Systemic Development}

The major human system groupings are: cardiovascular, coelomic cavity, endocrine, gastrointestinal tract, genital, head, immune, integumentary, musculoskeletal, neural, neural crest, placenta, renal, respiratory, and sensory. Human embryo stage events identified for anatomical systems are linked out to content providing an overview of that system's development. In this way each content page is limited in overall scope, length, and content to focus on a specific topic. These system pages, and most online topic pages, have a common structure: introduction, current research, developmental timeline, movies, animal model comparisons, molecular topics, abnormalities, selected references, terms, and external links. Text is minimised on the page, balanced with research images and data, and can be further linked. All online images can be opened separately and provide additional descriptive information, links to the original research articles, and copyright information.

The page header is collapsible and caters to many different language users. The online content is designed in the English language, but also through the page header allows an external Google translation of the content into 27 different languages. Once a translation has been selected and carried out, all internal links also then automatically go to a translated version in the same language. While these automatic translations are not completely accurate, in recent years they have improved substantially.

The page footer shows how to correctly cite the page and other human embryology content that links to that current page. In terms of cross-referencing, beneath the footer the extremely useful tool of "category" can be found.

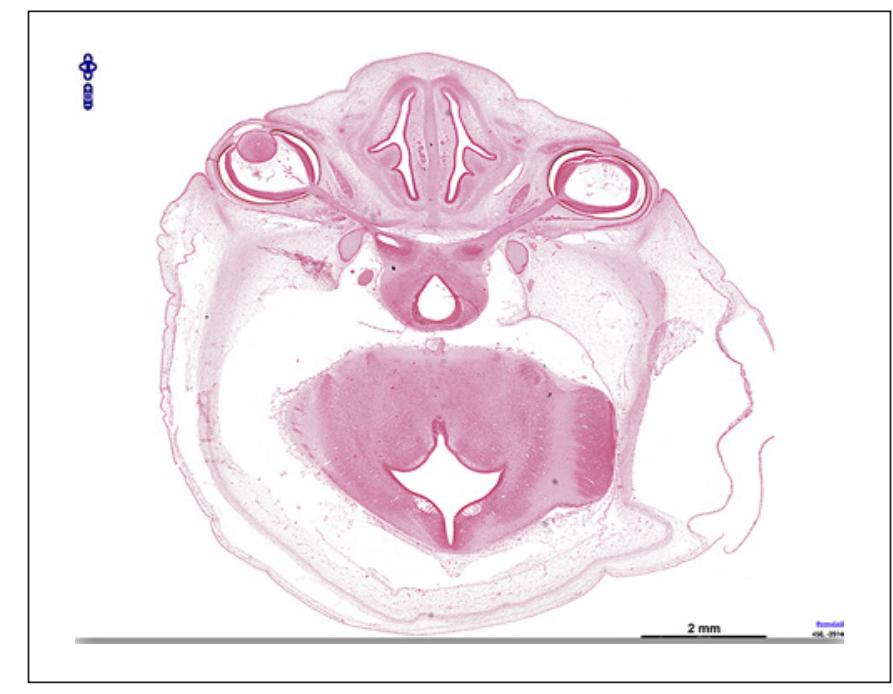

Fig. 2. This figure shows a screenshot of a human embryo virtual slide of Carnegie stage 22 (transverse section) through the head region at the level of the developing eyes (http://tiny.cc/Stage_22_ Eyes). The tools shown at the top left of the image allow online adjustment of the magnification and position of the histology section. The "Permalink" function at the bottom right of the image allows the user to generate an HTLM link to the current view and position of the image. This link can be copied and pasted into other pages or sent as an email to other users. Note that this is the Embryology website virtual slide implementation (http://tiny.cc/ Virtual_Slides) and not the DEC database OMERO version.

Selecting a listed category will open that category page which lists all local content, terms, pages, images, and movies that share this category link. Furthermore, the linked images are shown in an easy to view thumbnail version.

\section{Developmental Dynamics}

Human development is about the differentiation changes in cells, tissues, and organs over time. The best way to demonstrate these dynamic processes and events for both embryology education and research is through movies (http://tiny.cc/Embryo_Movies). Image animations simplify complex embryo morphological changes, for example in fertilisation, implantation, and cardiogenesis. Research microscopy movies, both bright field and fluorescent, have become more common in live human fertilisation and the animal model developmental literature. These movies are valuable tools to understanding and directly visualising developmental processes, often using molecular labels, and are timestamped. An example 
of human fertilisation, timestamped from zona pellucida penetration through to early blastocyst division, is included. The later process of human blastocyst development from morula through to blastocyst hatching is also shown. In collaboration with the Kyoto collection, as previously discussed regarding Carnegie stages, high-resolution MRI and EFIC imaging of the later human embryo stages (week 4-8) are included [Krishnan et al., 2014]. These movies show human embryo surface views (head, trunk, and limb development), neural and ventricular development, and internal organ and skeleton relationships. A number of clinical collaborators have provided deidentified ultrasounds that show examples of commonly occurring congenital abnormalities. These human abnormalities include: ectopic implantation, cleft lip and palate, spina bifida, gastroschisis, and a range of heart and placental abnormalities.

\section{Congenital Abnormalities}

Clinical embryology focuses on understanding the development of human congenital abnormalities of genetic, environmental, and unknown causes. The current research techniques include animal models and the identification of new molecular markers. An excellent online text database of human genetic abnormalities is already available - Online Mendelian Inheritance in Man (OMIM, https://www.omim.org) - which cross-references genes, rare genetic diseases (Orphanet, https://www.orpha.net), research history, clinical trials, and related molecular databases. The equivalent animal abnormality database, covering the vertebrate animals, is the Online Mendelian Inheritance in Animals (OMIA, http://omia.org).

The human embryology website does not replicate these existing database resources, though it does link to these and other databases on the appropriate abnormality pages. There is an attempt to include genetic and environmental teratogens that impact upon normal human development and when this occurs, including the concept of "critical periods of development." Many teratogenic factors have already been previously identified and even their synergistic interactions are reasonably well known. The online site recognises that the modern dynamic environmental changes may introduce teratogenic changes in the prevalence of, or even novel, developmental abnormalities. Some environmental changes include: maternal age, maternal nutrition, maternal physiology, new industrial compounds, new drugs, pathogens, and even environment change.

Two Web Resources Linking Major Human Embryology Collections Worldwide

\section{Digital Embryology Consortium}

The second part of this report provides an initial description of the new online human embryology resource made by researchers for researchers, the DEC (https:// human-embryology.org). Historically, embryologists began in the late 19th and early 20th century with small numbers of human embryos collected ethically from maternal deaths, ectopic pregnancies, and many spontaneously aborted abnormal embryos. These embryos were organised into developmental sequences and subsequently serial sectioned and histologically stained for analysis. The embryos were the key to our understanding of the timeline of human prenatal development.

In the USA, Minot established the Harvard Collection and later, in 1914, Franklin Mall established the Carnegie embryo collection from this and the Chicago and Johns Hopkins University collections. This collection continued to grow with contributions to become the first large collection of normal and abnormal human embryos [Mall and Meyer, 1921]. This historic collection of embryos continues today to contribute to current embryology research. In Europe, there were a number of existing historic collections (Hubrecht, Hill) [Richardson and Narraway, 1999] and several new collections established from the early 20th century onwards. In Spain, the Orts-Llorca, Javier Puerta, and Domenech-Mateu collections were established in Madrid and Barcelona, respectively [Arechaga et al., 2009]. In Germany, the Blechschmidt and Hinrichsen collections were established, the latter also being used for scanning electron micrographic studies of human development. In the UK, the Hamilton-Boyd collection of embryos and placentas was established during the 1950s and 1960s, and today, in the 21st century, the new $\mathrm{HuDSeN}$ project [Kerwin et al., 2010] and the Human Developmental Biology Resource [Gerrelli et al., 2015] are available. In Japan, during the 1960s, Hideo Nishimura established the Kyoto collection [Nishimura et al., 1968; Yasuda, 2018], which has since grown to over 44,000 human specimens, the largest collection of human embryos in the world today [Yamada et al., 2018].

Materials within the historic collections are still actively contributing to current embryological research, and the following recent references represent some examples from a few of the different collections: Arraez-Aybar et al. [2008], Hikspoors et al. [2016], Mekonen et al. [2017], Miyazaki et al. [2017], Okumura et al. [2017], RodriguezVazquez et al. [2018], Tojima et al. [2018], Warmbrunn et al. [2018]. There are few modern human embryo collections. Of the few, the $\mathrm{HuDSeN}$ project has been established mainly for molecular analysis, and to collabora-

Cells Tissues Organs 2018;205:293-302 297 
tively distribute both histological material and cells to researchers [Gerrelli et al., 2015].

The problem today is that many of these historic collections can only be accessed by researchers located at the institutions where the collections are held. Some of the earliest histological material also range from more than 100 years ago and are rapidly deteriorating. Visiting researchers and institutes have previously digitised parts of their collections, and some of this data is available through either publications or limited online access. There was no co-ordinated program of collection digitisation and preservation, and such a process is both expensive and difficult to fund through the existing research grant processes. In addition, much of the photographic or scanning process was often carried out before the current high-resolution slide scanning, databases, and storage tools software and technologies were available.

In 2014, following discussion with researchers and collection curators, the DEC (https://human-embryology.org) was established. The objective of this international partnership is "to digitise, preserve, and make available for researchers the major embryology histological collections."

\section{Digitisation}

Digitisation of slides uses a Zeiss AxioScanZ1 scanner with slide carrier adaptors that allow batch scanning of several historic slide sizes. After slides are loaded into carriers there is no further physical handling of the slides during the scanning process, minimising any damage to material. Serial sectioned embryos are then scanned at a resolution equivalent to $20 \times$ optical resolution. The scanner provides automatic as well as manual setting of multiple focus points, which is critical for the older slide materials. The digital files can be provided in a variety of formats, including an uncompressed format, although a single uncompressed section image can more than $4 \mathrm{~Gb}$ in size. The slide scanner is physically relocated to each holding institution to minimise any collection transport damage and the holding institutional policies concerning collection use. In 2015 scanning commenced with the Blechschmidt collection, which was completed in 2016.

\section{Preservation}

Most historic human collection histological stains have been stable for extended periods, with slides generally stored in the dark. The most visible loss of staining occurs at the side edges with oxidation or reduction. An additional problem is that while the stain may fade, the historic mounting media gain colour and yellow, and also suffer shrinkage cracking with age. It is unlikely that these historic human slides will ever be unmounted and restained. Researcher handling and transport of the slides has also contributed to damage or loss of collection material. An additional problem with the slides several collections was the great variety of slide sizes and thicknesses that existed before the standardised sizes we have today. This slide size issue also limited the scanning options available.

The digital version of collection slides preserves the material in its current state, protecting against any future deterioration. The availability of a digital version also means that the original material does not require unnecessary access and can be better conserved. Initially, there will be at least three versions of each collection's digital slides stored physically in different locations. The collection institute will hold a complete digital copy of all scanned material in their own requested digital format. The DEC co-ordinator will hold a second physical copy on duplicate drives. The third copy will be located on the online database server drives.

The current online server is running in a secure environment on an Apple Quad-Core Intel Xeon E5 platform. Data storage is on an external thunderbolt 2 connected RAID5 formatted 48 TB HDD stack. This storage space can be easily expanded, and the entire server hardware is easily relocated if required.

All scanned slides are stored as originally named in the collection, or by the current collection curator's data format. The online database uses the JPG XR image format that reduces storage requirements by approximately $80 \%$. This size reduction comes with minimal loss of resolution; for example, within scanned and compressed images individual cell mitotic profiles can still be identified. Each scanned slide file includes a low-resolution overview image of the entire slide, an image of the slide label region, and an individual high-resolution image (ROI) for each section on the slide.

\section{Availability}

The DEC maintains any collection's existing copyright and reuse conditions, and agreeing to this condition is a requirement for any researcher accessing the online scanned material. Following agreement to the DEC conditions of use, the database is freely available for research- 




Fig. 3. Blechschmidt 11-mm embryo slide 60 in the DEC embryo database opened within a web browser window. The OMERO interface has a three-panel format. The left-hand panel shows the available slides. The centre panel shows the individual slide images: label photograph, low-resolution whole slide image, and individual ROI sections from the slide. The right-hand panel shows embedded metadata and editable tags that can be added to the slide or individual sections. In this last panel the chain link in the upper right-hand corner can be used to share a link to other registered users of this specific view (http://149.171.80.223:8080/webclient/?show= dataset-641). The Blechschmidt Collection images (Fig. 3-5) are reproduced with the permission of Prof. Christoph Viebahn, director of the Institute of Anatomy and Embryology, University Medical Center, Göttingen.

ers to access on any internet-connected computer using any web browser (Fig. 3-5). The Blechschmidt Collection images are reproduced with the permission of Prof. Christoph Viebahn, director of the Institute of Anatomy and Embryology, University Medical Center Göttingen. Note these three figures are screenshot images as shown on a web browser, reduced in size and quality, and not accurately representative of original image resolution. The DEC website is accessible to all users, though no database material is available to unregistered users and all database activities are logged.

Two Web Resources Linking Major Human Embryology Collections Worldwide
The key to easy online collection researcher availability is a stable, well-supported image database environment. DEC selected the Open Microscopy Environment Remote Objects (OMERO, https://www.openmicroscopy.org/omero) software platform [Allan et al., 2012; Eliceiri et al., 2012]. The DEC server is currently running OMERO version 5.4. This software is a widely supported package that enables online access to, and analysis of, biological data in many different image formats. The database can be accessed not only through any web browser, but also securely through some image analysis software packages (see below). 


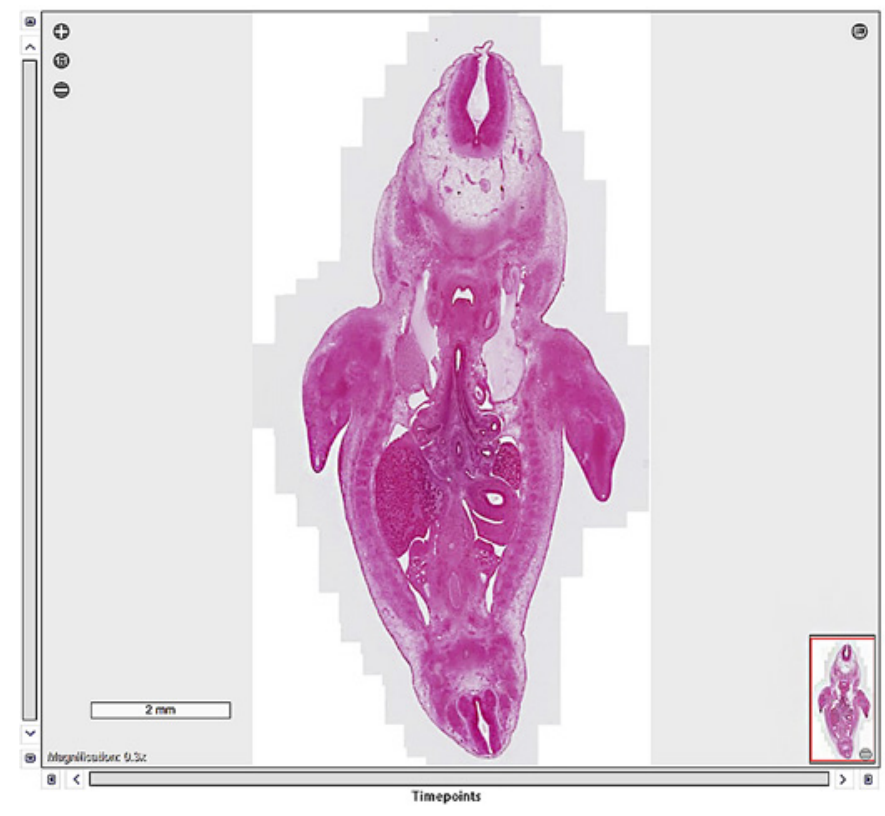

Fig. 4. The Blechschmidt 11-mm embryo Scene \#0 shown in Figure 3. The overall size of this single ROI is X: 26112px Y: 50880px. The original ROIs can be opened at full resolution directly within Fiji for analysis or $3 \mathrm{D}$ reconstruction from the individual serial sections. Overlays can also be generated and re-uploaded into the database as separate layers.

There is also the ability for users to add their own and share metadata tags and analysis to individual or sets of images. Image analysis and measurements can be made within the database, or through the Fiji (https://imagej. net/Fiji) distribution of the ImageJ software [Schindelin et al., 2012]. This external form of image analysis also allows re-uploading of the original image, preserving any additional layers that may have been added during analysis in this software.

The database currently contains an initial set of test images from the Blechschmidt, Hinrichsen, and $\mathrm{Hu}-$ brecht collections. Each uploaded image is meta tagged with the original collection information. This tagging ensures that any embryo images sourced and used by a researcher can be correctly attributed and comply with reuse conditions of that specific collection. Registered users can also be provided access to upload their own previously scanned human embryo images in a wide range of formats. Within the database, when any image is opened and viewed at any magnification, a unique HTML link is generated. This HTML link can be copied and sent to other researchers, which will allow them to have an identical image view within their own browser. These links can also be embedded within any online web pages.



Fig. 5. The Blechschmidt 11-mm embryo Scene \#0 shown in Figure 4 . This is a detailed view of a single bronchial epithelium within the developing left lung. Note the epithelium organisation and the mitotic profiles of dividing cells visible as condensed chromatin.

The project has now completed the scanning of two collections (Blechschmidt, Hinrichsen) and commenced the third (Hubrecht and Hill). The online human embryology database is currently focussed upon histological embryo imaging, although capable of accepting and storing other human data forms. These additional imaging formats include, but are not limited to: fluorescent, lightsheet, photographic, X-ray, MRI, EFIC, CT, ultrasound, doppler ultrasound, animations, and $3 \mathrm{D}$ reconstructions.

The DEC hopes that by making this human histological material more widely available, researchers with new methods of analysis can develop new insights into and understanding of human development. This also opens the opportunity for new 3D reconstruction and virtual reality methods of representation of these embryos. Such a vision for understanding embryology was part of Blechschmidt's original concept when he developed reconstruction models of embryos nearly a metre high.

The DEC is also prepared to accept contributions from external users and new institutional partners of their existing digital images or membership to the consortium. The consortium held an opening meeting in Göttingen (April 2016) and a second research meeting recently in Berlin (December 2017); the proceedings of this second 
meeting will be published at a future date. Dr. Mark Hill and Prof. Shigehito Yamada are the current contacts for further DEC information and contributions.

\section{Discussion}

Developmental biology research today is now exploring the minutia of molecular signalling and morphological events during embryogenesis. Nearly all of these current studies employ, through necessity, the use of animal models, and there are many online resources to support both researcher and educational understanding of animal developmental stages and features. There are far fewer online resources focussing on human development and no single online resource hopes to cover all aspects of embryogenesis. There are several alternative easily retrievable online resources. These alternate resources' scope and content can be classified into either static educational sites, histological sections, and reconstructions, or gene expression atlases. The Embryology website described in this report contains the broadest collation of historic understanding and the most current human research, in particular identifying timelines for whole human embryo, specific organs, and tissue development. The site has been continuously editorially updated for more than 20 years, providing a reference resource for both embryology researchers and educators.

The second online resource under development and identified in this report is the DEC. The members of this consortium have a common goal "to digitise, preserve, and make available for researchers the major embryology histological collections." It is also highly unlikely that these human embryo histology collections will ever be replicated in the future, and therefore represent a unique research resource. The DEC online resource will bring together the main human embryological collections from around the world. In the past each collection was available only at the site of the collection and to a small number of researchers. In addition, no comparison or correlation between human specimens in different collections could be easily made. The digitised specimens can also be specifically tagged, annotated, and labelled, which will allow database searching for common features and for comparison with human gene expression projects (HuDSeN project [Kerwin et al., 2010] and Human Developmental Biology Resource [Gerrelli et al., 2015]). The DEC histology database will also be a resource for the development of new virtual reality, augmented reality, and 3D printed human embryo models.

Two Web Resources Linking Major Human

Embryology Collections Worldwide
New online technologies have allowed the development of curated and readily accessible information on human embryonic development. While modern research techniques mainly employ animal models, we still require a detailed understanding of human development in many research and clinical areas, for example in assisted reproductive technologies, congenital abnormalities, and stem cell therapeutics. Human research material is by its nature ethically limited and the majority of histological material is contained within collections that are not easily accessible. The DEC will allow a new generation of developmental researchers not only access to these resources, but also the ability to build upon this invaluable resource themselves.

\section{Acknowledgement}

The Embryology website acknowledges the many other researchers, academics, clinicians, and students who have contributed to the development of these online resources, all of whom are specifically named online as a contributor. I especially thank Prof. Christoph Viebahn, director of the Institute of Anatomy and Embryology, University Medical Center Göttingen, for permission to use the Blechschmidt collection embryo screenshot images in this report. The Digital Embryology Consortium was supported by a UNSW Foundation grant for the slide scanner purchase and specifically thanks the Skalla family donation. Consortium members have to date received institutional support for their scanning programs. We are currently seeking financial support to complete this important project.

\section{Statement of Ethics}

The human histology collections described in this current paper are historically collected according to the national regulations relevant at that time and covered by ethics committee approvals from the originating institutions.

\section{Disclosure Statement}

The authors declare no conflicts of interest. 


\section{References}

Allan, C., J.M. Burel, J. Moore, C. Blackburn, M. Linkert, S. Loynton, D. Macdonald, W.J. Moore, C. Neves, A. Patterson, M. Porter, A. Tarkowska, B. Loranger, J. Avondo, I. Lagerstedt, L. Lianas, S. Leo, K. Hands, R.T. Hay, A. Patwardhan, C. Best, G.J. Kleywegt, G. Zanetti, J.R. Swedlow (2012) OMERO: flexible, model-driven data management for experimental biology. Nat Methods 9: 245-253.

Arechaga, J., J. Jimenez-Collado, D. Ruano-Gil (2009) A glance at Spanish embryology and teratology during the XX century through the academic life of Francisco Orts-Llorca (19051993). Int J Dev Biol 53: 1165-1177.

Arraez-Aybar, L.A., A. Turrero-Nogues, D.G. Marantos-Gamarra (2008) Embryonic cardiac morphometry in Carnegie stages 15-23, from the Complutense University of Madrid Institute of Embryology Human Embryo Collection. Cells Tissues Organs 187: 211-220.

de Bakker, B.S., K.H. de Jong, J. Hagoort, K. de Bree, C.T. Besselink, F.E. de Kanter, T. Veldhuis, B. Bais, R. Schildmeijer, J.M. Ruijter, R.J. Oostra, V.M. Christoffels, A.F. Moorman (2016) An interactive three-dimensional digital atlas and quantitative database of human development. Science 354: 6315.

de Bakker, B.S., K.H. de Jong, J. Hagoort, R.J. Oostra, A.F. Moorman (2012) Towards a 3-dimensional atlas of the developing human embryo: the Amsterdam experience. Reprod Toxicol 34: 225-236.

Dhanantwari, P., E. Lee, A. Krishnan, R. Samtani, S. Yamada, S. Anderson, E. Lockett, M. Donofrio, K. Shiota, L. Leatherbury, C.W. Lo (2009) Human cardiac development in the first trimester: a high-resolution magnetic resonance imaging and episcopic fluorescence image capture atlas. Circulation 120: 343-351.

Drake, R.L., J.M. McBride, N. Lachman, W. Pawlina (2009) Medical education in the anatomical sciences: the winds of change continue to blow. Anat Sci Educ 2: 253-259.

Eliceiri, K.W., M.R. Berthold, I.G. Goldberg, L. Ibanez, B.S. Manjunath, M.E. Martone, R.F. Murphy, H. Peng, A.L. Plant, B. Roysam, N. Stuurman, J.R. Swedlow, P. Tomancak, A.E. Carpenter (2012) Biological imaging software tools. Nat Methods 9: 697-710.
Gerrelli, D., S. Lisgo, A.J. Copp, S. Lindsay (2015) Enabling research with human embryonic and fetal tissue resources. Development 142: 3073-3076.

Hikspoors, J.P., H.K. Mekonen, G.M. Mommen, P. Cornillie, S.E. Kohler, W.H. Lamers (2016) Infrahepatic inferior caval and azygos vein formation in mammals with different degrees of mesonephric development. J Anat 228: 495-510.

Hill, M.A. (2007) Early human development. Clin Obstet Gynecol 50: 2-9.

Hill, M.A. (2018) Developing the Digital Kyoto Collection in Education and Research. Anat Rec 301: 998-1003.

Hill, M.A., K. Shiota, S. Yamada, C.W. Lo (2016) Kyoto Embryology Collection. Apple iTunes. https://itunes.apple.com/book/ id1143922693.

Kerwin, J., Y. Yang, P. Merchan, S. Sarma, J. Thompson, X. Wang, J. Sandoval, L. Puelles, R. Baldock, S. Lindsay (2010) The HUDSEN Atlas: a three-dimensional (3D) spatial framework for studying gene expression in the developing human brain. J Anat 217: 289-299.

Krishnan, A., R. Samtani, P. Dhanantwari, E. Lee, S. Yamada, K. Shiota, M.T. Donofrio, L. Leatherbury, C.W. Lo (2014) A detailed comparison of mouse and human cardiac development. Pediatr Res 76: 500-507.

Mall, F., A. Meyer (1921) Studies on abortuses: a survey of pathologic ova in the Carnegie Embryological Collection. Contrib Embryol 12: 364.

Mekonen, H.K., J. Hikspoors, G. Mommen, N. Kruepunga, S.E. Kohler, W.H. Lamers (2017) Closure of the vertebral canal in human embryos and fetuses. J Anat 231: 260-274.

Miyazaki, R., H. Makishima, J. Manner, H.G. Sydow, C. Uwabe, T. Takakuwa, C. Viebahn, S. Yamada (2017) Blechschmidt Collection: revisiting specimens from a historical collection of serially sectioned human embryos and fetuses using modern imaging techniques. Congenit Anom 58: 152-157.
Nishimura, H., K. Takano, T. Tanimura, M. Yasuda (1968) Normal and abnormal development of human embryos: first report of the analysis of 1,213 intact embryos. Teratology 1 : 281-290.

Okumura, M., A. Ishikawa, T. Aoyama, S. Yamada, C. Uwabe, H. Imai, T. Matsuda, A. Yoneyama, T. Takeda, T. Takakuwa (2017) Cartilage formation in the pelvic skeleton during the embryonic and early-fetal period. PLoS One 12: e0173852.

Richardson, M.K., J. Narraway (1999) A treasure house of comparative embryology. Int J Dev Biol 43: 591-602.

Rodriguez-Vazquez, J.F., M. Yamamoto, S. Abe, Y. Katori, G. Murakami (2018) Development of the human incus with special reference to the detachment from the chondrocranium to be transferred into the middle ear. Anat Rec 301: 1405-1415.

Schindelin, J., I. Arganda-Carreras, E. Frise, V. Kaynig, M. Longair, T. Pietzsch, S. Preibisch, C. Rueden, S. Saalfeld, B. Schmid, J.Y. Tinevez, D.J. White, V. Hartenstein, K. Eliceiri, P. Tomancak, A. Cardona (2012) Fiji: an opensource platform for biological-image analysis. Nat Methods 9: 676-682.

Tojima, S., H. Makishima, T. Takakuwa, S. Yamada (2018) Tail reduction process during human embryonic development. J Anat 232: 806-811.

Warmbrunn, M.V., B.S. de Bakker, J. Hagoort, P.B. Alefs-de Bakker, R.J. Oostra (2018) Hitherto unknown detailed muscle anatomy in an 8-week-old embryo. J Anat 233: 243-254.

Yamada, S., M. Nagai, M. Hagiwara (2018) The 40th anniversary of the Congenital Anomaly Research Center, Kyoto University Graduate School of Medicine. Anat Rec 301: 947-950.

Yamada, S., R.R. Samtani, E.S. Lee, E. Lockett, C. Uwabe, K. Shiota, S.A. Anderson, C.W. Lo (2010) Developmental atlas of the early first trimester human embryo. Dev Dyn 239: 1585-1595.

Yasuda, M. (2018) The beginning of Kyoto Collection: focus on memories. Anat Rec 301: 951-954. 